\title{
Apresentando o BEM: Um Objeto de Aprendizagem para mediar o processo educacional de crianças com deficiência visual e videntes nas operações básicas de Matemática.
}

\author{
André Luiz P. Dantas ${ }^{1}$, Gabriela R. P. Rezende Pinto ${ }^{1}$, Claudia Pinto P. Sena ${ }^{1}$ \\ ${ }^{1}$ Departamento de Exatas \\ Universidade Estadual de Feira de Santana (DEXA) - Feira de Santana, BA - Brasil \\ \{dededobem, gabrielarprp, caupinto.sena\}@gmail.com
}

\begin{abstract}
This article seeks to present the learning objects as an alternative educational resource to be used in the training process of visually impaired and sighted children. Moreover, it presents the BEM (Blind, Education and Mathematics), a learning object type electronic game designed to motivate them during the process of learning the operations of mathematics.

Resumo. Este artigo busca apresentar os objetos de aprendizagem como um recurso educacional alternativo a ser utilizado no processo de formação de crianças deficientes visuais e videntes. Além disso, apresenta o BEM (Blind, Education and Mathematics), um objeto de aprendizagem do tipo jogo eletrônico desenvolvido para motivá-las durante o processo de aprendizagem da operações básicas da Matemática.
\end{abstract}

\section{Introdução}

Os grandes avanços ocorridos na área das Tecnologias de Informação e Comunicação (TIC), como o surgimento do computador e o advento da internet, contribuíram significativamente para mudanças no paradigma pedagógico, isto é, na maneira como as pessoas ensinam e aprendem. Observa-se que as escolas buscam se informatizar e se apoiar em recursos provenientes das TIC para complementar o processo educacional e torná-lo mais interativo. Observa-se ainda uma transformação na forma como os materiais educacionais estão sendo produzidos e difundidos (WILEY, 2000).

Por outro lado, o mundo globalizado, interligado por tecnologias, também incita alguns desafios, dentre os quais se inclui a desigualdade social, agora intensificada pela exclusão digital. Os seres humanos necessitam desenvolver habilidades e competências relacionadas ao processamento de informações, que envolve a aquisição e uso de equipamentos, além das obtidas a partir da alfabetização, formação fundamental, média e universitária, para se prepararem para o exercício da cidadania e não se distanciarem do mundo tecnológico e comunicacional (BAUMAN, 1999).

Um desafio especial está relacionado à inclusão social de grupos discriminados (e.g. pessoas com algum tipo de deficiência, idosos, mulheres, menores infratores etc.) (CAZELOTO, 2008). A pessoa com deficiência visual, por exemplo, necessita de um acompanhamento educacional e social diferenciado, a fim de conseguir desenvolver suas habilidades e obter com isso uma condição de vida mais condizente com a sua 
realidade. Por isso, a Secretaria de Ciência e Tecnologia (SECT), pertencente ao Ministério da Ciência e Tecnologia (MCT), em parceira com o Instituto de Tecnologia Social (ITS), realiza uma pesquisa para acompanhamento de tecnologias que as auxiliam, chamadas de tecnologias assistivas. Esta pesquisa visa disponibilizar e acompanhar as atividades do uso das tecnologias assistivas nos diversos setores de produção da sociedade (RODRIGUES, 2010).

Dentre os tipos de tecnologia assistiva que vem sendo desenvolvidos para auxiliar as pessoas com deficiência visual, podem-se citar os softwares e as máquinas, criados para oferece-las opções de educação, interatividade, trabalho, lazer, entre outros. $\mathrm{Na}$ categoria software, o Objeto de Aprendizagem (OA) é um exemplo, que apesar de constituir uma proposta recente no sistema educacional brasileiro e também mundial (final da década de 1990), inúmeras publicações e conceitos sobre a temática já podem ser evidenciados (WILEY, 2000). Outro recurso eletrônico que pode motivar a aprendizagem e a socialização entre pessoas com deficiência são os jogos eletrônicos, cujos comandos são realizados através do áudio, ou seja, o jogador escuta as ações ocorridas no jogo e as responde por meio de um teclado de computador.

Diante do atual contexto social de produção e difusão/disponibilização de tecnologias assistivas, este trabalho objetiva contribuir com o processo de inclusão sociodigital a partir da concepção, desenvolvimento e disponibilização de um OA, que apresenta características de um jogo eletrônico, denominado Blind, Education and Mathematics (BEM). O BEM pode ser utilizado tanto por crianças com deficiência visual quanto por crianças videntes que estão em fase de desenvolvimento, e iniciando o processo de aprendizagem das operações matemáticas.

\section{Educação Matemática, Objetos de Aprendizagem e Jogos.}

Uma das possibilidades que a escola dispõe para auxiliar as crianças encontra-se na Educação Matemática. Para os Parâmetros Curriculares Nacionais (MEC, 1997), a Matemática tem o intuito de formar cidadãos, ou seja, preparar para o mundo do trabalho e ajudar na relação com as outras pessoas que vivem inseridas em seu meio social. Contudo, Rêgo e Rêgo (2000) destacam que se faz urgente a introdução de novas metodologias de ensino da Matemática, nas quais o estudante seja sujeito ativo da aprendizagem, respeitando-se o seu contexto e levando-se em consideração os aspectos recreativos e lúdicos das motivações próprias de sua idade, sua imensa curiosidade e desejo de realizar atividades em grupo.

Alguns dos recursos que vêm auxiliando a educação matemática são baseados em TIC, e destacam-se neste trabalho os Objetos de Aprendizagem (OA) e os jogos eletrônicos educacionais, que buscam proporcionar ao estudante uma aprendizagem lúdica e divertida. Miranda (2004, p. 14) diz que os OA "são elementos de um novo tipo de ensino baseado no computador e na Internet, fundamentado no paradigma de Orientação a Objetos da Ciência da Computação". Para Sosteric e Hesemeier (2002), são arquivos digitais (imagens ou filmes, por exemplo) que podem ser utilizados com fins educacionais e que incluem, internamente ou através de ligação, sugestões sobre o contexto apropriado no qual deve ser utilizado. Bettio e Martins (2004) dizem que qualquer entidade digital que tenha a capacidade de exprimir algum conhecimento pode ser considerada um OA. Já Spenelli $(2005$, p. 7) diz que "um objeto virtual de 
aprendizagem é um recurso digital reutilizável que auxilie na aprendizagem de algum conceito e, ao mesmo tempo, estimule o desenvolvimento de capacidades pessoais, como por exemplo, imaginação e criatividade". Outro conceito importante nesse cenário é o do Learning Technology Starndards Committee (LTSC), do consórcio Institute of Electrical and Electronics Engineers (IEEE), que define o OA como sendo qualquer material digital ou não digital que pode ser utilizado, reutilizado ou referenciado durante o ensino com suporte tecnológico (IEEE/LTSC, 2000). O BEM contempla alguns desses conceitos, especialmente a definição de OA proposta pela IEEE.

Assim, consideramos que um jogo eletrônico pode ser equiparado a um OA, pois pode facultar ao usuário a internalização de conhecimentos, contribuir significantemente para formação do indivíduo e também promover a motivação da aprendizagem. Através dos jogos, são exercitados aspectos físicos e mentais do indivíduo, podendo ser utilizado, portanto, para aprender, de forma prazerosa e interativa, a Matemática, a leitura, a escrita, trocar pontos de vista, explorar o mundo. Os jogos têm a capacidade de proporcionar grandes desafios e ambientes visualmente atrativos, com a utilização de sons e imagens, que visam motivar e estimular o interesse, a atenção, a concentração e a memória do jogador. Com tais características, eles deixaram de ser apenas instrumentos de entretenimento e passaram a se destacar como importantes recursos de apoio ao aprendizado (BROUGÈRE, 1998).

\section{Metodologia da Pesquisa}

A fim de sistematizar as atividades, a pesquisa aqui apresentada foi planejada em 3 etapas: Etapa 1 - Levantamento de informações; Etapa 2 - Desenvolvimento do Objeto de Aprendizagem BEM e a Etapa 3 - Verificação-Validação do Objeto de Aprendizagem BEM e Análise dos Resultados obtidos.

Na Etapa 1- Levantamento de informações foi realizada a maior parte da Pesquisa Bibliográfica. Buscou-se compreender o universo da criança (vidente e com deficiência visual): a educação Matemática voltada para tal público, o impacto do computador e dos recursos digitais em suas vidas e os OA, como uma possibilidade de recursos para motivá-las na aprendizagem das operações básicas de Matemática. Também foram analisados alguns jogos eletrônicos direcionados para as pessoas com deficiência visual, tais como o Dark Labyrinth (http://www.gamevicio.com) e Top Speed (http://www.playinginthedark.net). A partir de uma busca realizada nos Anais do XXII e XXIII Simpósio Brasileiro de Informática em Educação (SBIE), verificou-se que algumas experiências relacionadas à temática de modelagem e desenvolvimento de softwares educacionais; à inclusão sociodigital; e à concepção, desenvolvimento e aplicação de OA, também vêm sendo divulgadas e discutidas na comunidade (SILVA e PAULA, 2011; PIERINI et al., 2012).

A Etapa 2 - Desenvolvimento do Jogo foi dedicada ao desenvolvimento do jogo BEM. Primeiramente, foi realizado o levantamento dos requisitos funcionais e dos não-funcionais que o OA deveria apresentar. Em seguida, iniciou-se a modelagem do OA e a tomada de algumas decisões de projeto: que linguagem de programação seria utilizada, que software seria necessário para realizar a programação e também uma estimativa de quanto tempo levaria para finalizar todo o desenvolvimento. Os principais recursos utilizados no desenvolvimento foram: o Netbeans e o Eclipse, a linguagem de 
programação Java, a biblioteca Java Speech API e o software IBM ViaVoice. Também foram consideradas as recomendações da Engenharia de Software orientadas por Sommerville (2011), que adverte que Processos de Software são utilizados por quem deseja desenvolvê-los em tempo hábil, minimizando riscos e buscando maior qualidade. Assim, o pesquisador-desenvolvedor orientou-se por uma perspectiva de implementação de software denominada de Desenvolvimento Evolucionário, cujas atividades de especificação, desenvolvimento e validação são intercaladas. E, assim, um sistema inicial foi desenhado a partir de especificações de requisitos levantados.

\section{A Etapa 3 - Verificação e Validação do BEM e Análise dos Resultados}

Obtidos foi dedicada aos processos de verificação e validação do BEM, assim como à análise e discussão dos resultados obtidos. Os testes com o software foram realizados em dois lugares: uma Brinquedoteca e uma Fundação (entidade filantrópica). Tanto para o teste realizado na Brinquedoteca quanto para o teste realizado na Fundação, o objetivo inicial era escolher cinco crianças com a faixa etária entre 7 e 11 anos, tomando-se como critérios de escolha: ter interesse em participar do teste; possuir uma noção básica de operações matemáticas e uma boa interação com o computador. Contudo, no momento de realização dos testes, a Fundação se encontrava em período de volta às aulas e, portanto, foram selecionados para o teste 1 criança e 3 adultos. Por ser uma pesquisa realizada diretamente com os usuários, também se buscou, nesta etapa, a orientação de metodologia de pesquisa das Ciências Humanas. Optou-se por uma abordagem de pesquisa qualitativa, que, para Minayo (2001), trabalha com o universo de significados, motivos, aspirações, crenças, valores e atitudes, o que corresponde a um espaço mais profundo das relações, dos processos e nos fenômenos que não podem ser reduzidos a operacionalização de variáveis. Com relação às técnicas de coleta de dados foram utilizadas a observação e a entrevista semiestruturada (MINAYO, 2001). A análise dos resultados obtidos baseou-se no método de Análise de Conteúdo, de Bardin (1979), que é uma técnica de investigação que tem por finalidade a descrição objetiva e sistemática do conteúdo manifesto da comunicação.

\subsection{Aspectos Éticos da Pesquisa}

Salienta-se que este trabalho foi enviado ao Comitê de Ética em Pesquisa (CEP), da instituição de vínculo do pesquisador-desenvolvedor, e os dados foram coletados somente após a apreciação e aprovação do projeto pelos membros do comitê. Os aspectos éticos da pesquisa foram cumpridos conforme a Resolução 196/96, do Conselho Nacional de Saúde (CNS). Os compromissos éticos (e.g a informação dos riscos, benefícios e procedimentos da pesquisa; a garantia de anonimato e privacidade das crianças/adultos participantes; e a confidencialidade dos dados) foram explicitados no Termo de Consentimento Livre e Esclarecido (TCLE), que foi disponibilizado para os responsáveis legais das crianças e para os adultos, e no Termo de Assentimento, assinado pelas próprias crianças, durante o convite para a sua participação. Sempre que foi percebida qualquer alteração no comportamento das crianças, durante a realização do teste, houve uma pausa no jogo, e, então, buscou-se escutá-la e deixá-la à vontade para continuar ou não a atividade, respeitando a sua liberdade e a sua autonomia, conforme sugerido pela referida resolução. Por fim, que todo o trabalho foi realizado com a devida autorização dos responsáveis pelas instituições que serviram de campo de estudo: a Brinquedoteca e a Fundação de apoio às pessoas com deficiência visual. 


\section{Resultados Obtidos}

\subsection{Objeto de Aprendizagem BEM - Blind, Education and Mathematics}

O BEM consiste em um Objeto de Aprendizagem que possui um tabuleiro, composto por números dispostos em 5 colunas e 4 linhas. Esses números são gerados de forma aleatória assim que o usuário escolhe a opção de jogo que deseja jogar. As opções de jogo são: jogo de somar, jogo de subtrair, jogo de multiplicar e jogo de dividir. Para a escolha dessas opções de jogo, o usuário com deficiência visual deverá pressionar as teclas "CTRL + 1", "CTRL + 2", "CTRL + 3" e "CTRL + 4", caso deseje jogar o jogo de soma, subtração, multiplicação ou divisão, respectivamente. Ao mesmo tempo em que os números no tabuleiro são gerados é gerado também o valor que será calculado a partir da soma, subtração, produto ou divisão entre dois números, os quais deverão ser determinados pelo jogador, conforme Figura 1.

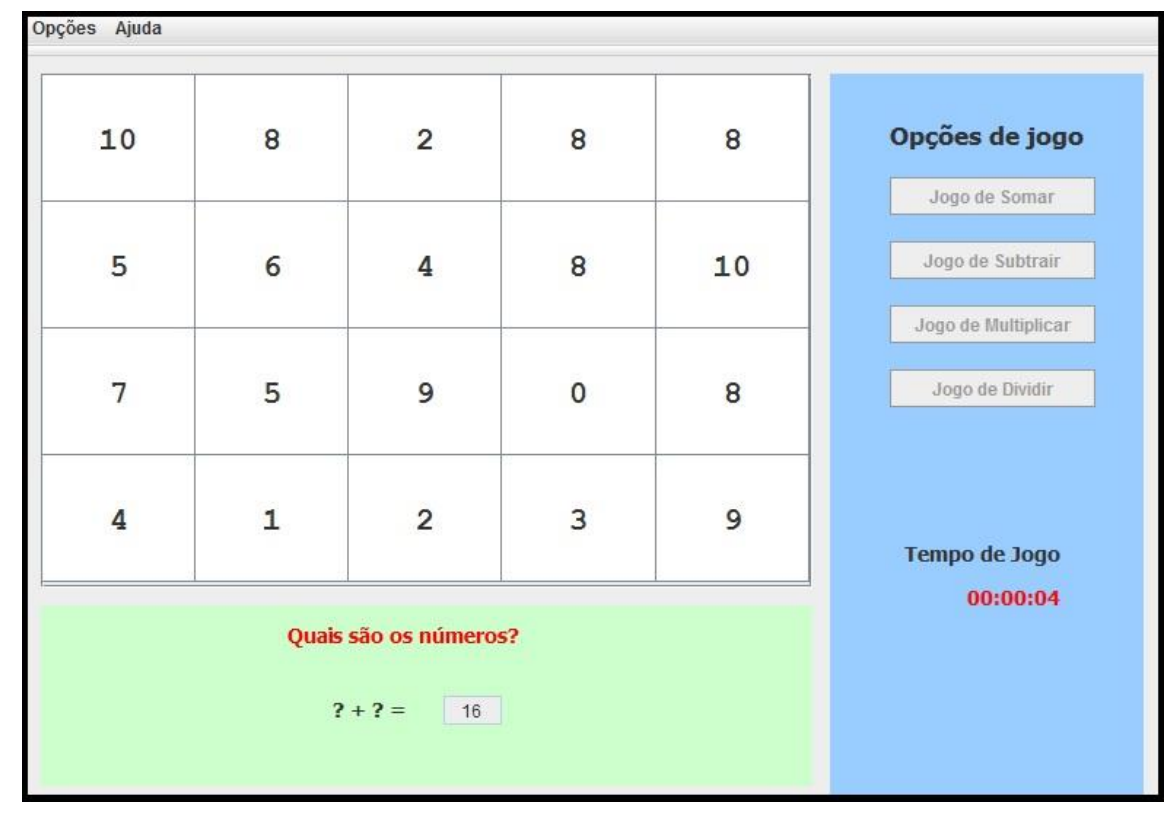

Figura 1 - Última versão executável do Jogo BEM. Fonte: Próprio Autor, 2013.

O usuário deverá escolher dois valores, dentro do universo de números dispostos no tabuleiro, valores esses que serão o resultado que irá satisfazer o valor do cálculo que foi gerado ao iniciar o programa. À medida que o usuário for percorrendo o tabuleiro, através do acionamento das teclas "up", "down", "left" e "right" do teclado, vai sendo retornado para o mesmo o som do número presente na célula que está selecionada. Conforme o jogador for encontrando os valores corretos, os números vão se apagando do tabuleiro. O objetivo principal do jogo é excluir todos os números presentes nesse tabuleiro no menor tempo possível, já que existe um cronômetro, que calcula o tempo de cada operação (i.e somar, subtrair, multiplicar ou dividir). Por se tratar de um OA voltado para videntes e pessoas com deficiência, além de possuir uma interface gráfica clara e amigável, todas as ações realizadas pelo jogador são sintetizadas por voz e retornadas para o mesmo. Por conta disso, existe um menu que possui um manual com todas as informações do jogo, no qual estão presentes as teclas de atalho que são utilizadas, bem como as suas regras e os seus objetivos. 


\subsection{Verificação e Validação do Objeto de Aprendizagem BEM}

Após finalização do desenvolvimento do jogo BEM foram realizados testes de verificação-validação com o público alvo especificado. Para auxiliar o pesquisadordesenvolvedor durante a observação, os seguintes critérios foram escolhidos: tempo de jogo, habilidade com o computador e com o BEM, habilidade com a Matemática, motivação ao utilizar o OA BEM. As Seções 4.2.1, 4.2.2 e 4.2.3 apresentam os resultados obtidos nos testes.

\subsubsection{Testes com os usuários com deficiência visual}

ParticipanteDV1 tem 8 anos, apresenta uma limitação visual em ambos os olhos, a qual foi adquirida por lesão na região ocular em função de cicatrizes de coriorretinite, com diagnóstico de cegueira. Apresentou dificuldades em entender os objetivos do jogo, não obtendo muito êxito em sua manipulação. Levou cerca de dezenove minutos para finalizar o jogo de soma. Apresentou habilidade na manipulação do computador. $\mathrm{O}$ desafio encontrado por esta criança na utilização do BEM foi a sua pouca compreensão em relação às contas de Matemática. Necessitou de muito auxílio para jogar e descobrir os números das contas que estavam sendo solicitadas. Apesar da experiência da criança com as contas de Matemática ser baixa e estar ainda em sua fase inicial, ela se mostrou motivada, sobretudo quando conseguia acertar os números que eram solicitados pelo jogo. Afirmou que conseguiu compreender todos os sons emitidos pelo BEM, e que ele pode sim lhe auxiliar no aprendizado das operações matemáticas.

ParticipanteDV2 é um adulto de 28 anos. Possui atrofia no nervo ótico e apresenta uma acuidade visual bastante limitada. Apresentou habilidade com o computador. Afirmou não utilizar muito jogos eletrônicos para treinar as operações matemáticas, pois utilizava apenas instrumentos como o sorobã. Apresentou dificuldade com as operações de Matemática, necessitando, em alguns momentos, de auxílio. Levou cinquenta e seis minutos e trinta e dois segundos para finalizar o jogo de somar. Mostrou-se motivado ao longo da atividade. No fim de sua participação, o usuário classificou o jogo como ótimo e afirmou que pode muito lhe ajudar com as operações matemáticas, sobretudo por ter confessado que o BEM foi para ele uma nova possibilidade de treinar e também aprender através do som e não apenas pela escrita.

ParticipanteDV3 é um adulto de 41 anos. Possui retinose pigmentar, apresentando baixa visão acentuada em ambos os olhos. Afirmou não possuir muita habilidade com o uso do computador. Apresentou algumas dificuldades, sobretudo com a prática do teclado, e, consequentemente, obteve um rendimento baixo no quesito de usabilidade do jogo. Também apresentou dificuldades em lidar com as operações de Matemática. Levou cerca de quarenta minutos para finalizar o jogo de somar. Mostrouse motivado a descobrir os números pedidos. Por fim, considerou que o jogo BEM pode lhe ajudar a treinar as operações matemáticas, e que também serve para estimular o jogador a raciocinar, sendo uma possibilidade a mais para acréscimo de conteúdo, no qual tem a chance de aprender brincando, auxiliado por um instrumento informatizado.

ParticipanteDV4 tem 35 anos. Este, também apresentando retinose pigmentar, com diagnóstico de cegueira, possui habilidade em manusear o computador. Levou cerca de oito minutos para finalizar o jogo de somar. Conseguiu estabelecer diversas estratégias para percorrer de maneira eficiente o tabuleiro presente no jogo. Não apresentou dificuldades de entender os objetivos do jogo e, por conseguinte, jogar de 
forma satisfatória. Apresentou habilidade com as operações matemáticas. Por conta de o participante ter afirmado não se interessar tanto por jogos eletrônicos, sua motivação foi contida. Além disso, caracterizou o jogo como sendo fácil, porém destacou que este pode sim contribuir para auxiliar no processo de aprendizagem das operações básicas e que também pode ser caracterizado como desafiador, pois estimula o jogador a pensar.

\subsubsection{Testes com os usuários videntes}

ParticipanteV1 tem 10 anos, e encontra-se no quinto ano do ensino fundamental, possui habilidade na manipulação do computador e não apresentou dificuldades ao utilizar o Jogo BEM. Levou cerca de seis minutos e três segundos para finalizar o jogo de somar, seis minutos e seis segundos para finalizar o jogo de subtrair e, por fim, quatro minutos e vinte segundos para concluir o jogo de multiplicar. Notou-se habilidade com a Matemática, resolvendo os cálculos de maneira bastante ágil. Também apresentou entusiasmo durante a experiência, sobretudo, por ter confessado estar utilizando um tipo de jogo, que disse nunca ter usado. Respondeu positivamente quando foi perguntado se o jogo poderia lhe ajudar nas operações básicas de Matemática.

ParticipanteV2 tem 8 anos, está cursando o terceiro ano do ensino fundamental. Apesar de possuir facilidade com o uso do computador, apresentou dificuldades no momento que precisava descobrir os dois números para completar a jogada. Levou doze minutos e vinte e três segundos para finalizar o jogo de soma. Apresentou pouca habilidade com as operações matemáticas. Ela afirmou não possuir muito interesse pela disciplina. Mostrou-se interessada, demostrando concentração e esforço para utilização do jogo de somar. Afirmou que conseguiu jogar de forma satisfatória e que o jogo poderá muito lhe auxiliar não só na prática, mas também na aprendizagem das operações de matemática, caracterizando o jogo como interessante.

ParticipanteV3 tem 7 anos, está cursando apenas o segundo ano do ensino fundamental. Mostrou as dificuldades no jogo de multiplicar. Segundo Marques e Silveira (2011), autores de livros de Matemática do ensino fundamental, a criança nesta fase estuda apenas as contas de somar e de subtrair. Possui habilidade com o computador. A dificuldade ocorrida foi mesmo em relação aos obstáculos que encontrou para lidar com as operações matemáticas. A participante apresentou-se motivada durante a atividade, mas achou o jogo difícil, sobretudo pelo fato de ainda estar aprendendo as operações matemáticas de somar e de subtrair.

ParticipanteV4 tem 10 anos, está iniciando o quinto ano do ensino fundamental e demonstrou bastante entusiasmo na hora de usar o jogo. Possui habilidade com o uso do computador. Obteve uma excelente desempenho ao jogar o BEM. Concluiu a partida em cinco minutos e cinquenta e cinco segundos, a de subtrair fez em cinco minutos e cinquenta e seis segundos e a de multiplicar finalizou em apenas cinco minutos e quarenta e dois segundos. Demonstrou afinidade pela Matemática. Conseguiu jogar de forma satisfatória, com motivação e compreendendo todos os detalhes de sua interface, tanto visual, quanto sonora.

ParticipanteV5 tem 9 anos, está no quinto ano do ensino fundamental. Obteve os melhores resultados nos testes. Para o jogo de somar levou apenas três minutos e quarenta e cinco segundos para concluir, no jogo de subtrair demorou cerca de três minutos e cinquenta e quatro segundos, e no jogo de multiplicar, finalizou em três minutos e vinte e dois segundos, somente. Explicou que a Matemática é a sua matéria 
preferida e que sempre possuiu grande facilidade para lidar com ela. Também afirmou possuir grandes facilidades em lidar com o computador, assegurando que já possui experiências com jogos eletrônicos e que utiliza a máquina por pelo menos três vezes na semana. Afirmou que o jogo poderia lhe ajudar mais ou menos, justificando, logo em seguida, que se trata de um jogo fácil, podendo ser melhorado em relação a sua dificuldade, acrescentando níveis a mais, como números dentro de uma faixa maior.

\subsubsection{Possibilidades e Desafios do Objeto de Aprendizagem BEM}

Durante os testes realizados com o BEM, alguns desafios puderam ser notados: todas as crianças entrevistadas (com deficiência visual e videntes) afirmaram nunca terem acesso a algum tipo de jogo eletrônico nas aulas de Matemática. Percebeu-se, ainda, que alguns fatores influenciam diretamente a utilização do jogo, tais como: o desenvolvimento cognitivo em que cada criança se encontra, ou seja, em que série ou em que idade a criança se encontra (o que pode levar a criança achar o BEM fácil ou difícil); o interesse maior ou menor pela Matemática e a habilidade em lidar com o computador.

A condição de cada criança também pode influenciar no custo inicial de aprendizagem do BEM. Nos primeiros minutos do jogo, especialmente para as pessoas com deficiência visual, era perceptível a dificuldade encontrada na utilização do BEM. À medida que jogavam, as estratégias iam sendo traçadas e o tabuleiro ia sendo conhecido. Ainda que considerado para algumas delas (principalmente para aquelas que já adquiriram habilidade com as operações matemáticas) como um jogo fácil, para as pessoas com deficiência visual o grau de dificuldade pode ser observado pelo maior tempo necessário para finalização de cada opção do jogo.

Ficou claro com a experiência que, conforme afirma Reily (2004, p. 60), "sem recursos especiais, estudantes com deficiência visual terão bastante dificuldade de acompanhar a matéria nas primeiras séries do ensino fundamental, bem como a partir da $5^{\text {a }}$ série, quando as exigências começam a aumentar". E, conforme constatado pela pesquisa, dos três indivíduos com deficiência visual que participaram dos testes do sistema, apenas um possuía um domínio mais acentuado das operações matemática, mesmo todos tendo já cursado completamente o período de educação escolar.

Segundo Vygotsky (1994), a cegueira cria dificuldades para a participação em muitas atividades da vida social e pedagógica. Warren (1994) complementa a ideia apresentando um enfoque comparativo, no qual apresenta uma comparação entre o desempenho médio de grupos de cegos e videntes. Os resultados, em geral, trazem médias mais baixas para os grupos de cegos, comparados aos de videntes, e esses resultados são tomados como indicação de limitações correlacionadas à cegueira.

A utilização do BEM também apresentou possibilidades, entre as que mais se destacaram, estão: a de contribuir para o desenvolvimento de habilidades computacionais; para potencializar a aprendizagem e prática das operações básicas de Matemática; para o desenvolvimento do raciocínio lógico e para uma aprendizagem lúdica e prazerosa.

Silva e Paula (2011), no trabalho "Utilização de jogos para pessoas com necessidades educativas especiais: uma análise experimental", também destacam a importância dos jogos educacionais digitais para o apoio e manutenção do aprendizado de pessoas com deficiência, pois tem o objetivo de motivar e estimular o interesse, a atenção, a concentração e a memória do usuário, contribuindo assim para uma maior 
fixação dos conteúdos que estão sendo trabalhados. Pierini et al. (2012) em seu trabalho intitulado "Brinquedos Numéricos: um jogo para o ensino dos conjuntos numéricos", apresenta "a concepção e o desenvolvimento de um jogo educativo computacional com o propósito de auxiliar no ensino e aprendizagem de conjuntos numéricos". O BEM vai ao encontro dessas propostas, e busca contribuir para uma educação inclusiva, já que pode ser utilizado por crianças com deficiência visual e por crianças videntes.

Quanto aos aspectos técnicos, verificou-se que o OA BEM atendeu aos seguintes requisitos levantados na Etapa 2 da pesquisa: RF01 - O usuário deverá ser capaz de realizar a escolha de dois números inteiros entre 0 e 10 , presentes em um tabuleiro, para que possam ser utilizados como resposta de uma dada soma, subtração, multiplicação ou divisão; RF04 - O OA retornará para o usuário, através da síntese de voz, todas as ações realizadas por ele e todas as informações necessárias para sua utilização; RF05 - Deverá ser cronometrado o tempo total de cada partida; RF06 Deverá ser retornado ao usuário o tempo total que levou para completar a partida; RF07 - O OA dará a possibilidade de ser manipulado através da utilização de teclas de atalho. Verificou-se, ainda, que dois requisitos funcionais não foram implementados: RF02 - O OA deverá registrar o nome do usuário para que sua pontuação possa ser armazenada após finalização do jogo; RF03 - O OA deverá possuir diferentes níveis de dificuldades.

Verificou-se, ainda, que os seguintes requisitos não-funcionais foram atendidos: RNF01 - O OA BEM deverá ser compatível com todos os sistemas operacionais pelo fato de ser desenvolvido pela linguagem de programação java, a qual é multiplataforma; RNF02 - A interface será simples e amigável, podendo ser utilizada tanto por usuários videntes quanto por não videntes; RNF03 - O jogo deverá ser implementado utilizando um padrão de programação orientado a objetos; RNF04 - O jogo deverá atender aos requisitos de eficiência, processando as ações dos usuários em tempo hábil.

\section{Considerações Finais}

Este artigo buscou apresentar o jogo BEM (Blind, Education and Mathematics), um objeto de aprendizagem do tipo jogo eletrônico desenvolvido para motivar crianças com deficiência visual e videntes no processo de aprendizagem das operações matemáticas.

Além de apresentar o BEM, o artigo mostrou os resultados que foram obtidos a partir dos testes que foram realizados para a sua verificação-validação. Verificou-se que o OA representa uma opção satisfatória para potencializar a aprendizagem de operações básicas de matemática, o raciocínio lógico, a motivação do estudante, as habilidades computacionais e que contribui para uma educação inclusiva.

Como trabalho futuro, implementar-se-á os requisitos funcionais RF02 - O OA deverá registrar o nome do usuário para que sua pontuação possa ser armazenada após finalização do jogo e o RF03 - O OA deverá possuir diferentes níveis de dificuldades.

\section{Referências}

Bauman, Z. (1999). "Globalização". Rio de Janeiro: Jorge Zahar.

Bardin, L. (1979). "Análise de conteúdo". Lisboa: Edições 70, 229 p.

Bettio, R. W. de; Martins, A. R. (2004). "Objetos de Aprendizagem: Um novo modelo direcionado ao Ensino a Distância”. Disponível em: <http://noticias.universia.com.br/destaque/noticia/2004/12/17/493047/bjetosaprendiz 
ado-um-novo-modelo-direcionado-ao-ensino-distncia.html>. Acesso em: $07 \mathrm{dez}$. 2011.

Brougère, G. (1998). "Jogo e educação”. Porto Alegre: Artes Médicas, 224 p.

Cazeloto, E. (2008). "Inclusão Digital: uma visão crítica”. São Paulo: editora Senac, São Paulo.

IEEE (2000). "Learning Technology Standards Committee (LTSC). Draft Standard for Learning Object Metadata". Institute of Electrical and Electronics Engineers, Inc. LTSC. Learning technology standards committee website. Disponível em: <http://ltsc.ieee.org/>. Acesso em: 06 dez. 2011.

Marques, C.; Silveira (2011) E. Matemática $2^{\circ}$ Ano. São Paulo: Ed. Moderna.

Mec (1997). "Parâmetros Curriculares Nacionais ( $1^{\mathrm{a}}$ a $4^{\mathrm{a}}$ série): matemática". Secretaria de Educação. Educação Fundamental. Brasília: MEC/ SEF.

Mec (2012). "Rived”. Disponível em: http://rived.mec.gov.br/site_objeto_lis.php. Acesso em: 26 jun. 2012.

Minayo, M. C. S. (2001). "Pesquisa social: teoria, método e criatividade". Rio de

Janeiro.

Miranda, R. M. (2004) "GROA: Universidade Federal do Rio Grande do Sul. Um gerenciador de repositórios de objetos de aprendizagem”. 80p, il. Tese (Mestrado).

Pierini, L. M. et al. (2012). "Brinquedos Numéricos: um jogo para o ensino dos conjuntos numéricos". In: SBIE 2012- XXIII Simpósio Brasileiro de Informática na Educação. Rio de Janeiro - RJ.

Rêgo, R.G.; Rêgo, R.M. (2000). "Matemática ativa”. João Pessoa: Universitária/UFPB, INEP, Comped.

Reily, L. (2004). “Escola Inclusiva: Linguagem e mediação”. Campinas: Papirus, (Série Educação Especial).

Rodrigues, R. P (2010). "Uma análise ontológica de jogos direcionados a pessoas com necessidades visuais: fase de preparação". Trabalho de Conclusão de Curso de Engenharia de Computação, Universidade Estadual de Feira de Santana.

Silva, R. A., Paula, M. M. V (2011). "Utilização de jogos para pessoas com necessidades educativas especiais: uma análise experimental”. In: SBIE 2011-XXII Simpósio Brasileiro de Informática na Educação. Aracaju - SE.

Sommerville, Ian (2011). "Engenharia de Software". São Paulo: Pearson Prentice Hall.

Sosteric, M.; Hesemeier, S. (2002). "When is a Learning Object not an Object: A first step toward a theory of learning objects". International Review of Research in Open and Distance Learning. Disponível em: <http://www.irrodl.org/index.php/irrodl/article/view/106>. Acesso em: 07 dez. 2011.

Spenelli, W. (2005). Aprendizagem Matemática em Contextos Significativos: Objetos Virtuais de Aprendizagem e Percursos Temáticos. Dissertação (Mestrado em Educação) - Universidade de São Paulo. São Paulo.

Vygotsky, L. S. (1994). "The Blind Child". Trad. Adjunto de Eudes Fabri. Colaboração: Achilles Delari Jr. E Eugenio Pereira de Paula Jr. Disponível em: <http://pt.scribd.com/doc/16420054/Vigotski-A-crianca-cega-traduzido-por-AEFabri>. Acesso em: 13 dez. 2011, 11:22.

Warren, D.H. (1994). "Blindness and Children: an individual differences approach". EUA: Cambridge University Press.

Wiley, D. (2000). "Learning Object Design and Sequenceing Theory". Brigham Young University, Provo. 35 p. 